Provided for non-commercial research and education use. Not for reproduction, distribution or commercial use.

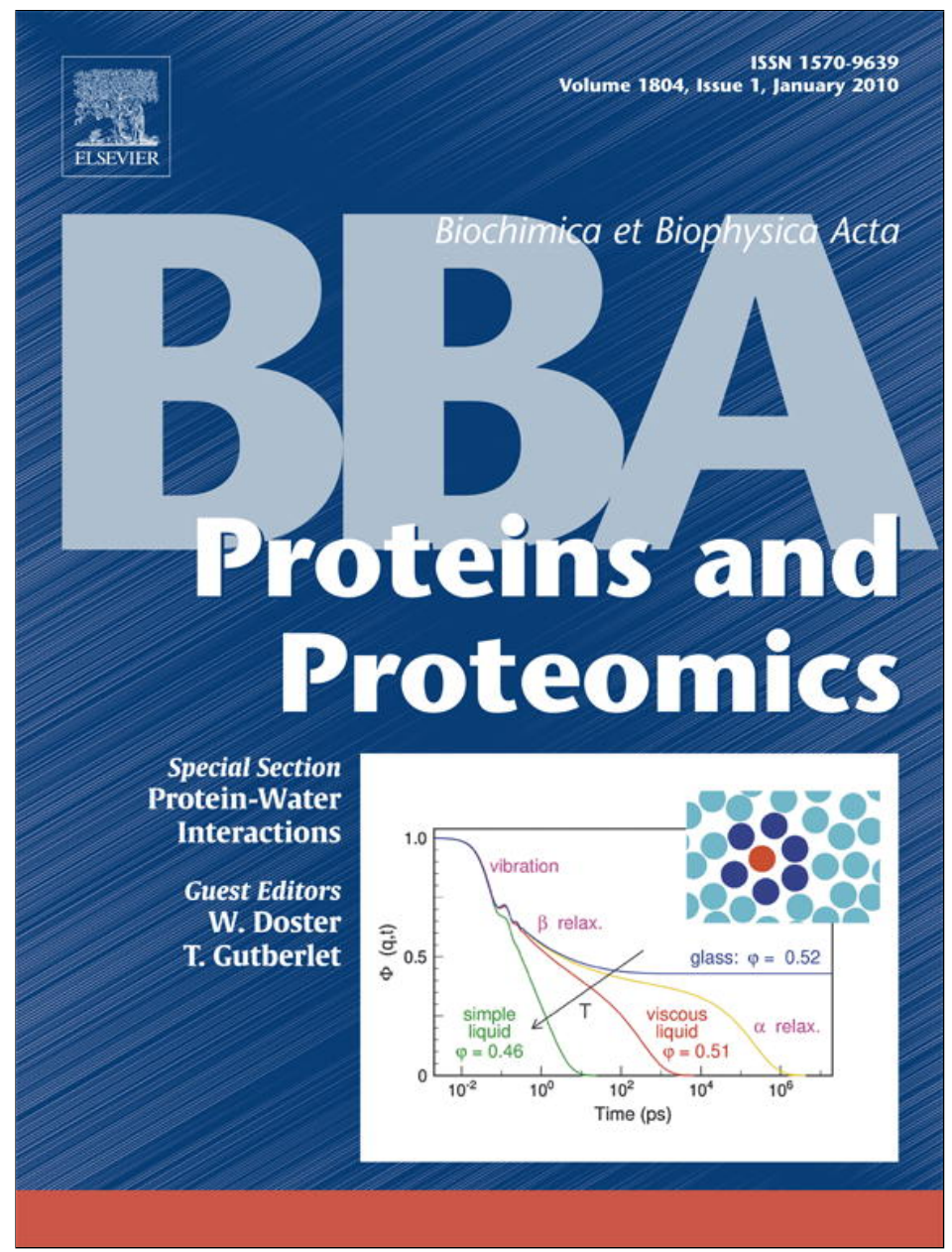

This article appeared in a journal published by Elsevier. The attached copy is furnished to the author for internal non-commercial research and education use, including for instruction at the authors institution and sharing with colleagues.

Other uses, including reproduction and distribution, or selling or licensing copies, or posting to personal, institutional or third party websites are prohibited.

In most cases authors are permitted to post their version of the article (e.g. in Word or Tex form) to their personal website or institutional repository. Authors requiring further information regarding Elsevier's archiving and manuscript policies are encouraged to visit:

http://www.elsevier.com/copyright 


\title{
The protein glass transition as measured by dielectric spectroscopy and differential scanning calorimetry
}

\author{
H. Jansson ${ }^{\mathrm{a}, *}$, J. Swenson ${ }^{\mathrm{b}}$ \\ a The Swedish NMR-centre at Göteborg University, SE-405 30 Göteborg, Sweden \\ b Department of Applied Physics, Chalmers University of Technology, SE-41296 Göteborg, Sweden
}

\section{A R T I C L E I N F O}

\section{Article history:}

Received 27 February 2009

Received in revised form 5 June 2009

Accepted 29 June 2009

Available online 10 July 2009

\section{Keywords:}

Protein

Glass transition

Dielectric spectroscopy

Differential scanning calorimetry

Protein dynamics

Solvent dynamics

\begin{abstract}
A B S T R A C T
The glass transition and its related dynamics of myoglobin in water and in a water-glycerol mixture have been investigated by dielectric spectroscopy and differential scanning calorimetry (DSC). For all samples, the DSC measurements display a glass transition that extends over a large temperature range. Both the temperature of the transition and its broadness decrease rapidly with increasing amount of solvent in the system. The dielectric measurements show several dynamical processes, due to both protein and solvent relaxations, and in the case of pure water as solvent the main protein process (which most likely is due to conformational changes of the protein structure) exhibits a dynamic glass transition (i.e. reaches a relaxation time of $100 \mathrm{~s}$ ) at about the same temperature as the calorimetric glass transition temperature $T_{\mathrm{g}}$ is found. This glass transition is most likely caused by the dynamic crossover and the associated vanishing of the $\alpha$ relaxation of the main water relaxation, although it does not contribute to the calorimetric $T_{\mathrm{g}}$. This is in contrast to myoglobin in water-glycerol, where the main solvent relaxation makes the strongest contribution to the calorimetric glass transition. For all samples it is clear that several proteins processes are involved in the calorimetric glass transition and the broadness of the transition depends on how much these different relaxations are separated in time.
\end{abstract}

(C) 2009 Elsevier B.V. All rights reserved.

\section{Introduction}

It is a well-known fact that the function of a protein is dependent on its internal motions. Also established is that its function, in some way, is related to its surrounding water, since it has been shown that proteins need a certain hydration level for full biological function [1], and that dry proteins are biologically inactive [2]. This can be explained by the plasticizing effect water has on proteins [3], i.e., the enhancement of the protein flexibility and motions necessary for its function. This clearly implies that the function of a protein is dependent on both its dynamics and its hydration. The question is, however, how the dynamics of a protein (and thereby its function) is related to its surrounding environment. Therefore, for a deeper insight into the biological function of a protein an understanding of the protein dynamics and how it is related to its surrounding environment is of fundamental importance.

The dynamics of protein hydration water has been the subject for many experimental studies. Several of these studies, performed by mainly dielectric spectroscopy, but also recently by nuclear magnetic resonance (NMR), see e.g. Refs [4-11], have shown that the temperature dependence of the relaxation time for the observed

\footnotetext{
* Corresponding author. Fax: +46 317863880

E-mail addresses: helen.jansson@chalmers.se, helen.jansson@nmr.gu.se (H. Jansson).
}

main process changes at a certain temperature (typically around $180 \pm 20 \mathrm{~K}$ ) from a low temperature Arrhenius behaviour to a nonArrhenius dependence at higher temperatures. The physical origin of this dynamic crossover is not fully established, and it has therefore been lively debated in the literature. Different interpretations have been discussed, for instance that it is caused by the glass transition of the protein-solvent system [12]. This interpretation is, however, contradicted by the fact that water confined in solid materials (where no glass transition of the system occurs at a similar temperature) also exhibits the same type of crossover [6,7]. Anyhow, irrespective of the exact physical origin of the dynamic crossover most results suggest that the process below the crossover temperature should be considered as a secondary process, distinguished from the cooperative and viscosity related $\alpha$-relaxation, which is unobservable with both dielectric spectroscopy and NMR techniques [10], at least below the crossover temperature [6,7]. If the $\alpha$-relaxation of the hydration water disappears, due to confinement effects, at the crossover temperature, it should have an impact on protein dynamics, and consequently also on protein function, since it has been shown that protein conformational changes [13,14], and escape of carbon monoxide (CO) out of the heme cavity in myoglobin [15], cannot occur without the viscosity related $(\alpha)$ relaxation in the surrounding solvent. Thus, in this case, such large scale protein fluctuations will disappear at the dynamic crossover temperature of the solvent, giving rise to a glass-like 
transition of the protein-solvent system. Below the crossover temperature the remaining secondary solvent process will only be able to promote more local protein motions [13]. In addition to this crossover also another dynamic crossover for hydration water, measured by quasielastic neutron scattering techniques, is observed around $220 \mathrm{~K}$ [16], but its physical nature will not be further discussed in this paper.

The dynamics of a protein is determined by its energy landscape [17]. The protein energy landscape, which describes how the energy of the protein is changed with change of its structure, can be compared to that of glass-forming liquids [17-19]. In this scenario, glasses are analogous to proteins at low temperatures, and supercooled liquids to proteins above a glass-like transition of the proteins, with the exception that for proteins the increase of fluctuations with increasing temperature is not directly caused by the increasing thermal energy. Rather the increase of protein fluctuations is caused by the increasing motions in the solvent [20]. This glass-like transition of proteins, which can be determined by calorimetry, should not be confused with the so-called "dynamical transition" of proteins that are probed by e.g. neutron scattering around $220 \mathrm{~K}$ [21,22] since these types of dynamical onsets (which occur whenever the dynamics become fast enough to be observed by the given experiment) are probed on very different time-scales. In general, for glass-forming materials the glass transition temperature $T_{\mathrm{g}}$ of a material is typically defined as the temperature where the viscosity exceeds a value of $10^{13}$ poise $\left(=10^{12} \mathrm{~N} \mathrm{~s} / \mathrm{m}^{2}\right)$ and/or the associated $\alpha$-relaxation time reaches the time-scale of a calorimetric measurement, i.e. about $100 \mathrm{~s}$ [23]. On this time-scale the viscosity related $\alpha$-relaxation and secondary $\beta$-relaxations are distinctly separated, whereas on the nano-second time-scale, which is the typical time-scale for a neutron scattering experiment, they are generally merged into one effective relaxation. This is an important difference, since for secondary relaxation processes the material behaves as a solid at $T_{\mathrm{g}}$ (or even slightly above $T_{\mathrm{g}}$ ) due to that the time-scale of the $\alpha$-relaxation is so much slower than these secondary processes, whereas at the time-scale of neutron scattering this is not the case.

Protein dynamics involve many different types of motions, both local and more global movements, including transitions between several conformational substates [13]. When lowering the temperature these transitions become increasingly slower with decreasing temperature, and at a certain temperature the protein becomes frozen in a specific substate [17,24]. The freezing of transitions between different conformational substates into a more rigid structure involves a change in the thermal energy of the protein, and consequently, a change in the heat capacity. As a result, the protein undergoes a glass transition. The glass transition in hydrated proteins has been investigated by calorimetric and rhehological measurements, see e.g. [25-29], and it has been shown that except for that this transition can be tricky to detect it is exceptionally broad and, thus, covers a large temperature interval [27-29]. The origin of this broadening has been suggested to be due to a large distribution of relaxation times within the protein-water system [27], or be caused by a size distribution of water clusters on the protein surface [29]. Thus, the glass transition in hydrated protein samples does not occur at one specific temperature or in a narrow range of temperatures, but rather this transition occurs over a very broad temperature interval. In the present study we have investigated hydrated myoglobin and myoglobin in a water-glycerol mixture by dielectric spectroscopy and differential scanning calorimetry (DSC). The aim of the study has been to relate the calorimetric glass transition in the proteinsolvent system to the relaxation processes obtained by dielectric spectroscopy. From our result we are able to show that the calorimetric glass transition is in fact due to several relaxation processes occurring on different time-scales.
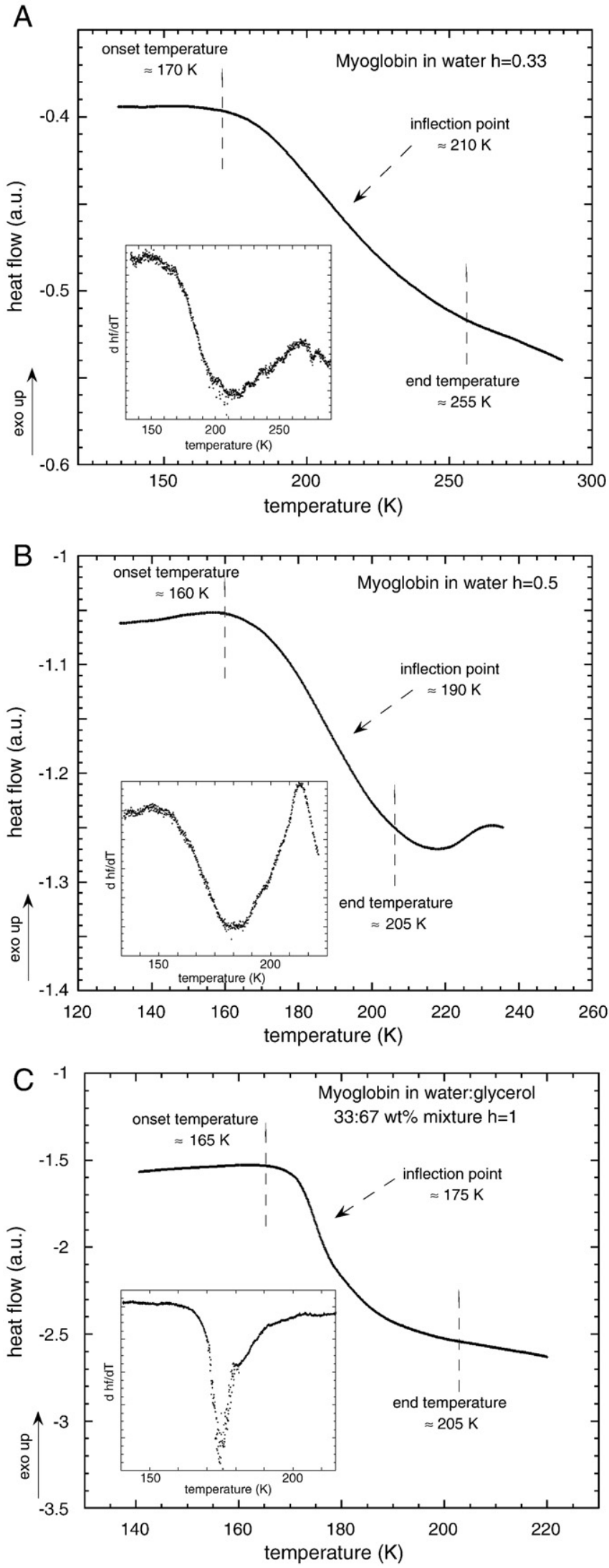

Fig. 1. DSC curves obtained for myoglobin in water at the hydration levels $h=0.33$ (A) and $h=0.5$ (B), and myoglobin in 33:67 wt.\% water:glycerol mixture (C) at the solvent level $h=1$. The insets show the derivative of the heat flow (hf) with respect to the temperature. 
A

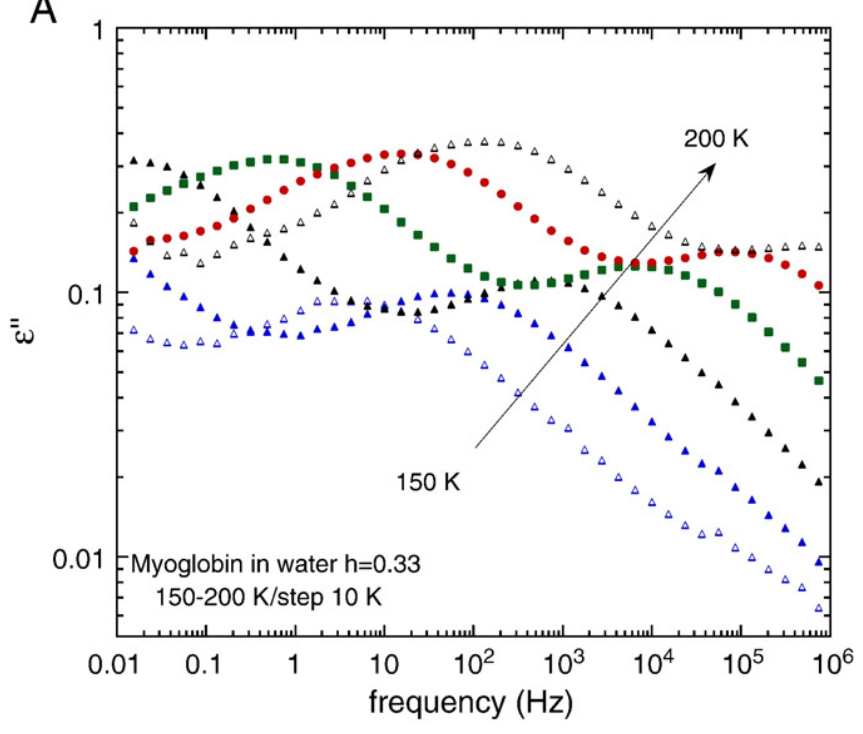

B

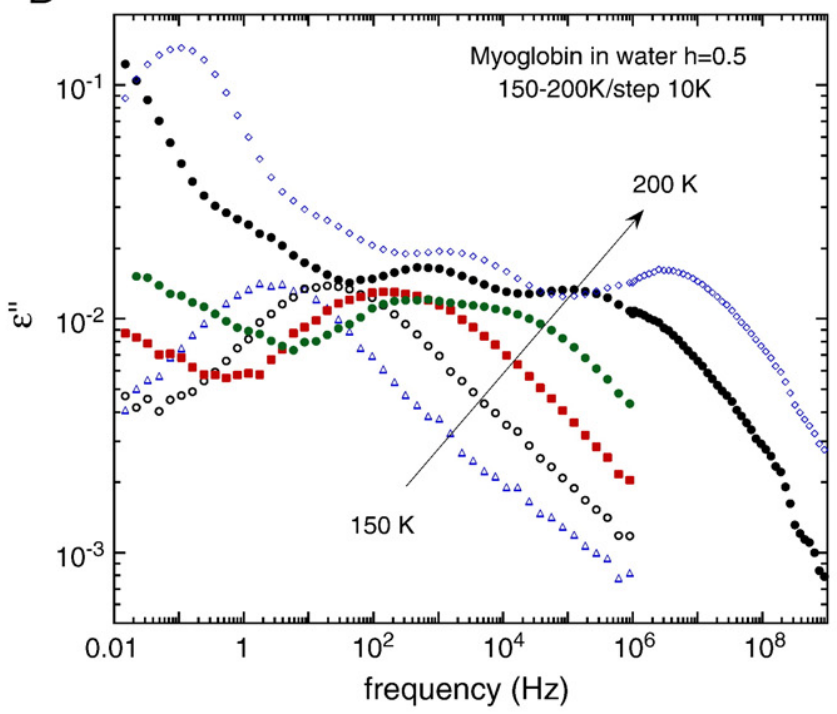

C

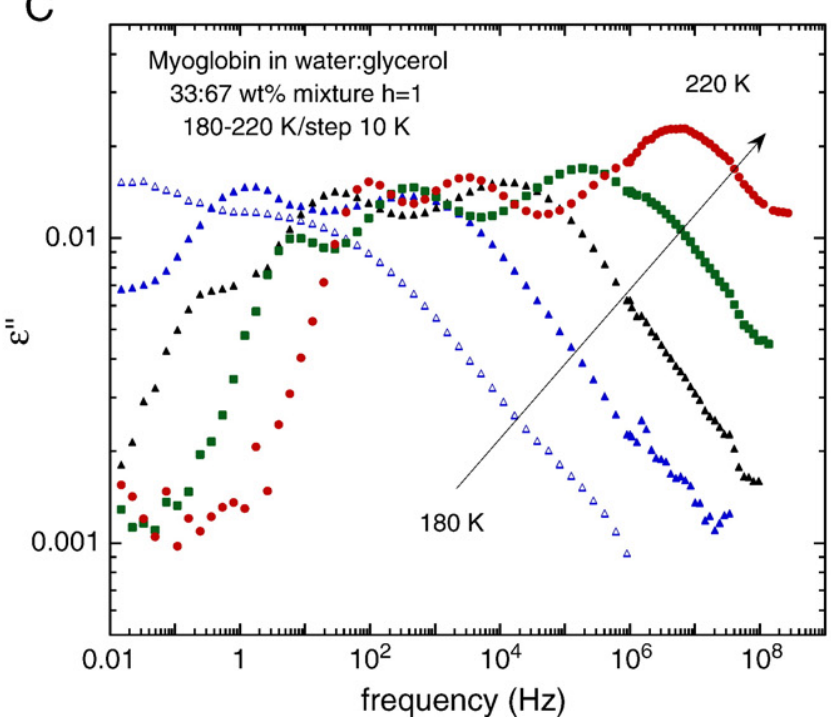

\section{Materials and methods}

The myoglobin used in this study was a horse heart (equine heart) myoglobin purchased from Sigma-Aldrich (product number M1882). The protein was in form of a freeze-dried powder, ( $\leq 10 \%$ water) and used without further treatment. The samples prepared for this study was protein in water at two different hydration levels $h=0.33$ and $h=0.5$ ( $h$ denotes the ratio of solvent to protein, i.e. $h=$ gram solvent/gram protein), and protein in a water:glycerol 33:67 wt.\% mixture of $h=1$, i.e. the latter sample contained equal amount of solvent and protein.

\subsection{Differential scanning calorimetry}

Differential scanning calorimetry (DSC) measurements were performed on TA Instruments DSC Q1000. For each measurement the sample $(\approx 5-10 \mathrm{mg}$ ) was placed in a hermetic pan of aluminium, and as a reference, an empty hermetic pan was used. All investigations were carried out in the heating mode in the temperature interval 95$295 \mathrm{~K}$, at a heating rate of $10 \mathrm{~K} / \mathrm{min}$ (after cooling down at the same rate). The temperature for the inflection point of the glass transition was determined using the Universal Analysis 2000 software (TA instruments), and the temperatures for the onset and the end of the transition were determined by analysing the derivative of the heat flow with respect to the temperature. Each measurement was carried out several times to ensure reproducibility of the results.

\subsection{Dielectric spectroscopy}

The dielectric measurements were performed on a broadband dielectric spectrometer from Novocontrol. The samples were investigated in the frequency and temperature ranges $10^{-2}-10^{9} \mathrm{~Hz}$ and $120-350 \mathrm{~K}$, respectively. For the lower frequencies $\left(10^{-2}-10^{7} \mathrm{~Hz}\right)$ an Alfa-S High Resolution Dielectric Analyser was used, and the measurements in the higher frequency range $\left(10^{6}-10^{9} \mathrm{~Hz}\right)$ were carried out using an Agilent 4291B RF Impedance Analyser. The samples were placed between two gold plated electrodes and a teflon film (thickness $100 \mu \mathrm{m}$ ), placed between the sample and the upper electrode, was used in order to reduce the large contribution of conductivity and electrode polarisation to the spectra at low frequencies (since this type of set-up has been shown to reduce such effects [30,31], even if care has to be taken when analysing the results [32]). The sample thickness was for all measurements $0.1 \mathrm{~mm}$ (determined by silica spacers), and the sample diameter was $20 \mathrm{~mm}$ and $10 \mathrm{~mm}$ for the low and high frequency measurements, respectively. After preparation each sample was placed in a sample holder and cooled down to $120 \mathrm{~K}$ and then reheated to $350 \mathrm{~K}$ while isothermal $( \pm 0.2 \mathrm{~K})$ scans were made at every fifth degree. The imaginary part of the dielectric response, $\varepsilon^{*}(f)=\varepsilon^{\prime}(f)-\varepsilon^{\prime \prime}(f)$ was then analysed. The dielectric loss peaks obtained for the protein sample were fitted to several Havriliak-Negami functions (Eq. (1)) and one more general fit function [33] (Eq.(2))

$$
\begin{aligned}
& \varepsilon^{\prime \prime}(\omega)=\sum \operatorname{Im}\left(\frac{\varepsilon_{s}-\varepsilon_{\infty}}{\left(1+(i \omega \tau)^{\alpha}\right)^{\beta}}\right) \\
& \varepsilon^{\prime \prime}(\omega)=\frac{\varepsilon_{p}^{\prime \prime}}{\frac{(1-c)}{a+b}\left[b\left(\omega / \omega_{p}\right)^{-a}+a\left(\omega / \omega_{p}\right)^{b}\right]+C}
\end{aligned}
$$

where $\omega=2 \pi f$ is the angular frequency. Specific parameters for the equations are in Eq. (1) the relaxation time $\tau$, the static dielectric

Fig. 2. Imaginary part of the dielectric spectra for myoglobin in water at the hydration level $h=0.33$ (A) $h=0.5$ (B), and in the 33:67 wt.\% water:glycerol mixture at the solvent level $h=1$ (C) for some temperatures. Note here that the low frequency dispersion caused by conductivity and polarisation effects is essentially absent in the data due to the use of a teflon film between the sample and one of the electrodes. 
constant and the limiting value of the dielectric constant at high frequencies $\varepsilon_{\mathrm{s}}$ and $\varepsilon_{\infty}$ respectively, and $\alpha$ and $\beta$ are shape parameters that determine the symmetric and asymmetric broadening of the relaxation peak, respectively. In Eq. (2) $\omega_{\mathrm{p}}$ and $\varepsilon^{\prime \prime}$ p are the position and the height of the peak. The parameters $a$ and $b$ are shape parameters that describe the slope of the peak at low and high frequency side, respectively, and the $C$ parameter is a parameter that describes the broadening of the relaxation peak without changing the power laws at high and low frequency sides.

The temperature dependences of the obtained relaxation processes were described by the Vogel-Fulcher-Tammann (VFT) equation (Eq. (3)) or by the Arrhenius law (Eq.(4))

$\tau=\tau_{0} \exp \left(\frac{-D T_{0}}{T-T_{0}}\right)$

$\tau=\tau_{0} \exp \left(E_{a} / k_{B} T\right)$

where $\tau_{0}$ is the relaxation time extrapolated to infinite temperature, $E_{\mathrm{a}}$ is the activation energy and $T_{0}$ the temperature where $\tau$ would go to infinity. The parameter $D$ determines the deviation from Arrhenius temperature dependence. Eqs. (3) and (4) are generally used to describe the $\alpha$ - and the $\beta$-relaxations, which are due to global configurational changes and more local movements in the material, respectively.

\section{Results}

The glass transition of a system can be observed experimentally by studying the thermodynamic properties of the system. In Fig. 1 the results from the DSC scans obtained for hydrated myoglobin at the two different hydration levels $h=0.33(1 \mathrm{~A})$ and $h=0.5(1 \mathrm{~B})$, and for myoglobin in the water:glycerol 33:67 wt.\% mixture at a total solvent level $h=1$ (1C) are shown. It has earlier been shown that the onset temperature of the glass transition in hydrated proteins is almost independent of the hydration level whereas the width of the transition decreases with increased hydration level [25]. In accordance with this, it is obvious that the reduction of the hydration level from $h=0.5$ (Fig. 3B) to $h=0.33$ (Fig. 3A) only has a minor effect on the onset temperature, whereas a substantial broadening of the glass transition region occurs from a width (as taken from the onset to the end temperature of the transition) of $45 \mathrm{~K}$ to a width of about $85 \mathrm{~K}$ for the higher compared to the lower hydration level, respectively. From these figures it is also clear that the glass transition temperature $T_{\mathrm{g}}$ (given by the inflection point) increases from about $190 \mathrm{~K}$ to around $210 \mathrm{~K}$, which is consistent with the plasticizing effect water has on the flexibility of proteins [3]. Keeping the water content at the lower hydration level and adding glycerol to the sample results in a more narrow glass transition and a lower $T_{\mathrm{g}}$, as shown in Fig. $1 \mathrm{C}$. In this case, where the solvent contains $67 \mathrm{wt} . \%$ glycerol, $T_{\mathrm{g}}$ is located at about $175 \mathrm{~K}$, and the width of the transition is reduced to $\approx 40 \mathrm{~K}$.

In Fig. 2 typical dielectric loss spectra are shown for some temperatures. From this figure it is clear that the spectra are complex, and that several relaxation processes are present in the data, but also that contributions from conductivity and electrode polarisation are suppressed due to the use of the teflon film between the sample and one of the electrodes. It should here be noted that some of these relaxation processes, i.e. the low frequency processes at higher temperatures, would not be visible without this teflon film, and thereby, much information about the relaxations in the sample had been lost. Hence, the teflon film made it possible to determine even processes that normally are hidden by the usually large contribution of conductivity and polarisation effects at low frequencies and high temperatures. The relaxation processes in the samples were determined by use of Eqs. (1) and (2), and in Fig. 3 the temperature dependences of all the obtained relaxation times $\tau$ are shown for
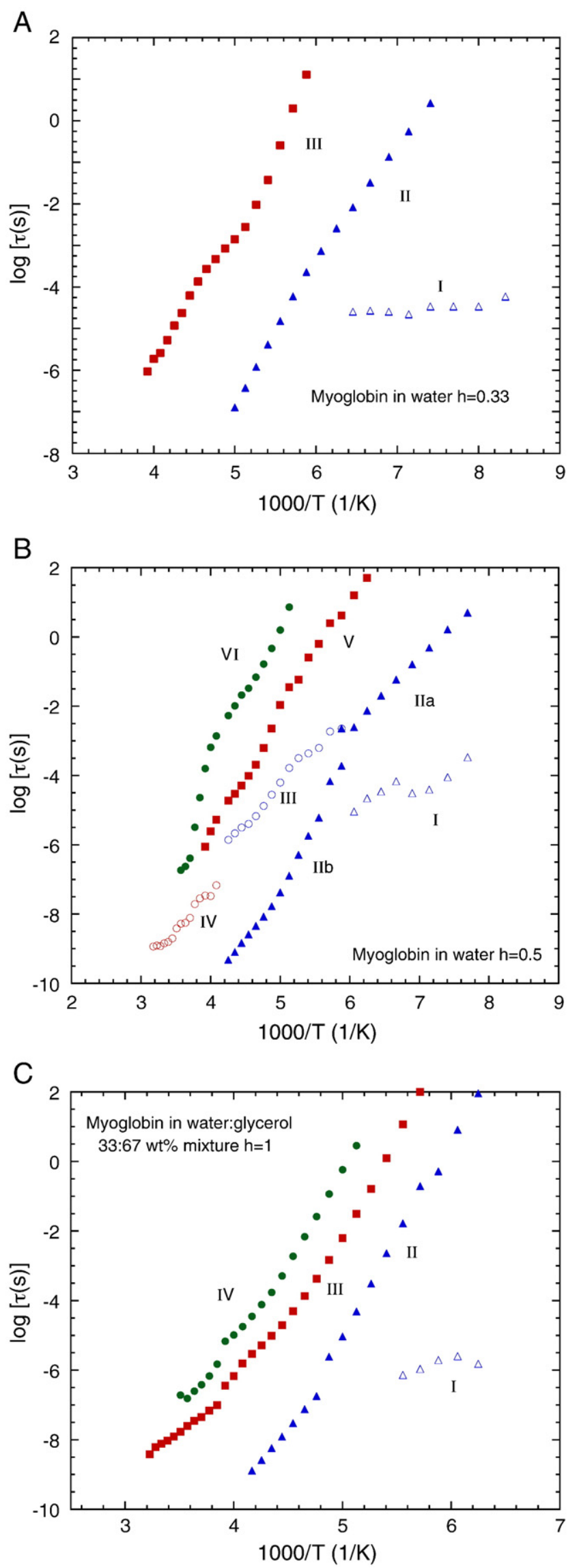

Fig. 3. Dielectric relaxation times obtained for myoglobin in water at the hydration level $h=0.33$ (A) $h=0.5$ (B), and in the 33:67 wt.\% water:glycerol mixture at the solvent level $h=1$ (C). 
myoglobin in water at the hydration levels $h=0.33$ (3A), $h=0.5$ (3B), and myoglobin in water:glycerol 33:67 wt.\% (3C), respectively. From these figures it is clear that the relaxation scenario of the sample at the highest water content $(h=0.5)$ is more complicated than the samples containing less water or a relatively large amount of glycerol. For these samples the fastest and second fastest relaxation processes (denoted process I and II) are due to the relaxation of the solvent, where process I is a weak local relaxation that appears to be universal for supercooled confined and hydration water. The universality of this process is supported by the fastest process shown in Fig. 4. The second fastest process, i.e. process II in Fig. 3, corresponds to the main relaxation of the solvent. In the case of pure water as solvent (Fig. $3 \mathrm{~A}$ and B), as well as water rich solvents ( $>50$ wt.\% water, H. Jansson, R. Bergman, and J. Swenson, unpublished data), this process is also rather universal for water supercooled in confinements, on surfaces and in mixtures, as also shown in Fig. 4. Its anomalous temperature dependence, with a crossover from a high temperature non-Arrhenius behaviour to a low temperature Arrhenius dependence at typically $180 \pm 20 \mathrm{~K}$, will be further discussed below. However, the data shown in Fig. 3B suggest that not all hydration water at higher hydrations undergo this crossover, but that a small fraction of it continues to relax by the secondary process (denoted process III in Fig. 3B) also above 180 K. Possible explanations for such a behaviour will also be discussed below. For the sample with 67 wt.\% glycerol in the solvent the interpretation of this main solvent process (process II) is considerably easier since it is due to the $\alpha$-relaxation of the mixed solvent (at least for temperatures down to $T_{\mathrm{g}}$ of the protein-solvent system).

In addition to the solvent processes (i.e. processes I and II) the samples exhibit one or more dielectric relaxation processes located at lower frequencies, see Fig. 3. In the case of the sample with $67 \mathrm{wt} . \%$ glycerol in the solvent (3C) the slowest of the processes (process IV) likely originate from large scale fluctuations in the protein, since this process corresponds, regarding relaxation times and temperature dependence, to results obtained by hole-burning spectroscopy concerning conformational changes of the protein structure [34]. However, since the corresponding bulk solvent also shows a similar slow relaxation process (provided that the teflon film is used also for that sample, H. Jansson, R. Bergman, and J. Swenson, unpublished data) we cannot exclude that it is due to collective motions in the solvent. Taken the preferential hydration into account, the second

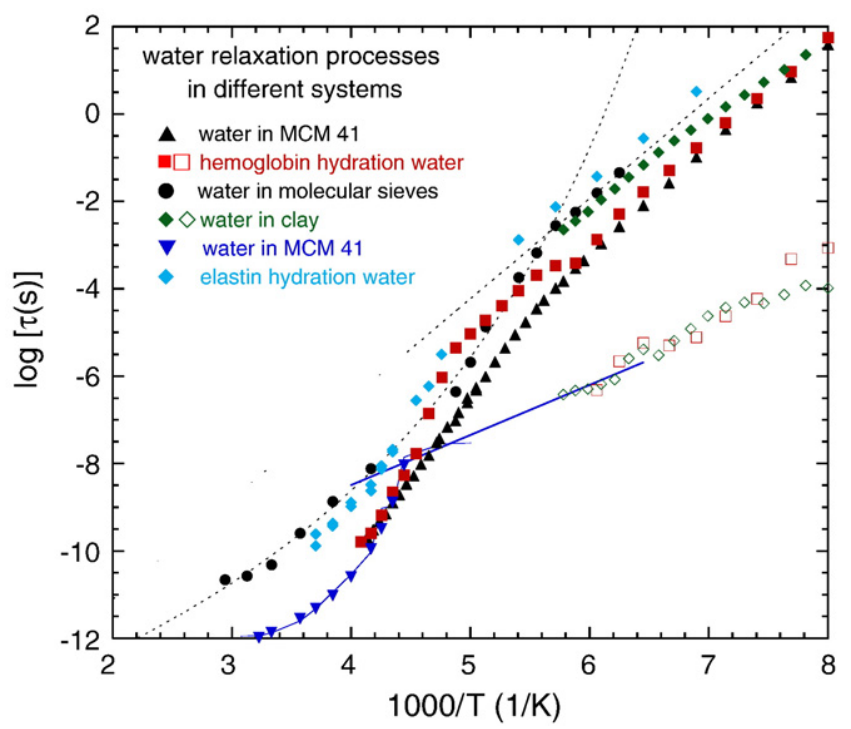

Fig. 4. Relaxation times for hydration water of different proteins $[5,10]$, and water confined in various hydrophilic host materials $[4,6,8,16,39]$. slowest process (process III, Fig. 3C) probably corresponds to process IV and process III in the myoglobin-water samples of the higher $(h=0.5$, Fig. 3B) and lower $(h=0.33$, Fig. 3A) hydration level, respectively. This relaxation process arises above the crossover temperature of the main water relaxation and it is most likely due to the relaxation of polar side groups based on earlier interpretations from measurements of hydrated proteins by time-domain reflectometry [35] and recent observations by quasielastic neutron scattering [36]. Whether these side chains move together with the possible secondary solvent relaxation (process III, Fig. 3B) is still not clear. In the myoglobin sample with the higher hydration level also two slower relaxation processes are observed, where the slowest one (process VI), in correspondence with the glycerol containing sample, most likely is due to large scale motions of the protein. A similar relaxation process was also found in the sample with the lower hydration level, but due to its weakness its temperature dependent characteristics is very uncertain, and it has, therefore, been excluded. The origin of process $\mathrm{V}$ in the $h=0.5$ sample is not known, but likely also this process corresponds to protein motions.

\section{Discussion}

As mentioned above, the main solvent process of myoglobin in pure water follows the same temperature behaviour as water in many different systems, both solid inorganic materials and biological and other soft systems, as shown in Fig. 4 and Refs [7,11]. In the case of soft systems the crossover from a VFT behaviour at higher temperatures to an Arrhenius temperature dependence at low temperatures is generally occurring at the glass transition temperature of the whole water containing system. It has therefore been suggested $[11,12]$ that it is the glass transition of the system that causes the crossover in the temperature dependence of the interfacial water, in one or another way. However, the same type of crossover occurs for water confined in solid materials, such as MCM-41 and molecular sieves shown in Fig. 4, for which a glass transition temperature is located far above the crossover temperature for the confined water. This makes it obvious that the glass transition of a soft system can only indirectly be responsible for the crossover. A likely explanation for the dynamic crossover in soft systems was provided by Cerveny et al. in Ref. [11], where the authors suggested that the crossover is associated with the emergence of confinement effects for the interfacial water when the host material starts to move above $T_{\mathrm{g}}$ of the system. Thus, below $T_{\mathrm{g}}$ of the system the water is restricted to move in confined spaces, whereas above $T_{\mathrm{g}}$ of the system the water molecules are able to perform cooperative long-range motions. In this way the authors were able to explain why the crossover occurs at $T_{\mathrm{g}}$ of soft systems without contradicting previous interpretations [6-8] of the dynamic crossover of water confined in solid materials. In these previous publications it has been suggested that the low temperature Arrhenius behaviour is due to a secondary $\beta$-like relaxation process of the deeply supercooled water, and that the crossover is a result of a merging of this secondary process with the cooperative $\alpha$-relaxation, which, for some reason, is non-observable with both dielectric spectroscopy and NMR techniques [10], at least below the crossover temperature. Thus, for water in both soft and solid materials the dynamic crossover seems to be caused by geometrical confinement effects.

It should here be noted that this main process of the dielectric and ${ }^{2} \mathrm{H}$ NMR relaxation data shown in Fig. 4, which we concluded must be due to the dynamics of the interfacial water since it is basically universal and present also in solid systems with only water molecules moving on the given time-scale, has also been suggested [37] to mainly arise from protein dynamics. Although fast local protein dynamics occurs on a similar time-scale as the main water process the small dielectric constant of a protein $(\varepsilon \approx 2-4)$ compared to that of water ( $\varepsilon \approx 80$ at room temperature) should ensure that the protein contribution to the dielectric relaxation process is minor compared to 
that of water. Thus, the fastest and most local protein relaxations are submerged in the main solvent process and only the slower and more global motions may be observable in Fig. 3.

As described above, the use of the teflon film made it easier to extract dynamical information of the systems that normally is hidden, or suppressed, by the contribution of conductivity and polarisation effects. In Fig. 3B, above the crossover temperature, a rather unexpected behaviour of the water relaxation is revealed. Whereas the main part of this relaxation seems to follow the, in general observed, VFT behaviour (process IIb), a smaller fraction of the water (process III) seems to continue the relaxation via an Arrhenius temperature dependence. This may imply that a fraction of the water displays a more local dynamics than the majority of the water molecules in this system, and recently an explanation of this behaviour was suggested [14] by that some of the water molecules found in the very close vicinity of the protein surface do not participate in the cooperative $\alpha$-relaxation and therefore continue to relax via the more local $\beta$-relaxation. However, a corresponding relaxation process in the sample at the lower hydration level ( $h=0.33$ ) is not observable, which may be due to that process III in Fig. 3B is directly or indirectly caused by the small amount of ice present in that sample. Thus, whether this process is due to ice or surface water on ice particles (as suggested in Ref. [38]) and/or protein molecules [14] is still not fully established.

For myoglobin in water the main solvent process, i.e. process II, reaches a relaxation time of $100 \mathrm{~s}$ at about $115 \mathrm{~K}$, which is far below the onset temperature of the broad $T_{\mathrm{g}}$ range. This observation further supports the assignment of this process to a secondary relaxation of the hydration water below the dynamic crossover temperature, since such a secondary solvent process is not expected to participate in the glass transition of the sample. The slowest process (process VI) shown in Fig. 3B, which most likely is due to large scale protein fluctuations since it is found very close to results obtained by hole-burning spectroscopy concerning conformational changes of the protein structure [34], should, however, be one of the main processes responsible for the glass transition of this sample, since this process reaches a relaxation time of $100 \mathrm{~s}$ at $185 \mathrm{~K}$, which is close to the inflection point around $190 \mathrm{~K}$ shown in Fig. 1B. In the case of pure water as the solvent the crossover in the water dynamics observed at about $170 \mathrm{~K}$ in Fig. 3A and B is close to the onset temperature of the glass transition (see Fig. $1 \mathrm{~A}$ and $\mathrm{B}$ ). This fact is most likely not a coincidence since the $\alpha$-relaxation of the hydration water vanish at the crossover temperature and no glass transition related conformational changes of the protein can occur without presence of the $\alpha$ relaxation in the solvent [13]. Thus, the crossover in the water dynamics, and the associated vanishing of the $\alpha$-relaxation, seems to cause the glass transition of the protein-solvent system and thereby have important implications for protein dynamics. However, since the interfacial water does not exhibit any clearly observable glass transition (due to this vanishing of the associated $\alpha$-relaxation before the calorimetric glass transition temperature is reached) the glass transition of protein-water systems is observed as a "freezing-in" of only protein motions. Nevertheless, the surrounding water is responsible for the glass transition of the protein-water system, as discussed above.

For the myoglobin sample in a mixed water-glycerol solvent, a somewhat different scenario for the glass transition is revealed. In this case the main solvent relaxation (process II in Fig. 3C), and the fastest clearly distinguishable protein process (process III in Fig. $3 \mathrm{C}$ ) reach a relaxation time of $100 \mathrm{~s}$ (i.e. a dynamical glass transition) at about $160 \mathrm{~K}$ and $174 \mathrm{~K}$, respectively, which is in almost perfect agreement with the onset temperature and the inflection point of the calorimetric $T_{\mathrm{g}}$ at $165 \mathrm{~K}$ and $175 \mathrm{~K}$, respectively, shown in Fig. 1C. From a comparison of the DSC data on the myoglobin sample in the mixed water-glycerol solvent (Fig. 1C) with the corresponding data on the myoglobin samples in pure water (Fig. 1A and B) it is evident that the step in $T_{\mathrm{g}}$ is larger and the inflection point is considerably closer to the lower end of the $T_{\mathrm{g}}$ range for the glycerol containing sample. This suggests that the freezing-in of the $\alpha$-relaxation in the water-glycerol solvent makes a major contribution to the calorimetric $T_{\mathrm{g}}$ of this sample, in contrast to the glass transition of the myoglobin samples in pure water where the main contribution, as discussed above, seems to arise from large scale conformational motions in the protein.

However, since the glass transition range is known to be exceptionally broad for proteins [27,28], particularly for the low hydrated samples shown in Fig. 1A and, it is clear that not only one single protein process can be responsible for the whole glass transition. Thus, the whole $T_{\mathrm{g}}$ range of a sample may involve the freezing-in of both the $\alpha$-relaxation of the solvent, if that is present at $T_{\mathrm{g}}$, as well as different types of protein fluctuations, and when these protein fluctuations occur on widely different time-scales the $T_{\mathrm{g}}$ range becomes particularly broad, as shown in Fig. 1A.

\section{Concluding remarks}

The present calorimetric and dielectric study of myoglobin in water and water-glycerol solvents shows how strongly the glass transition, and its associated dynamics, of the protein-solvent samples depends on the total solvent content and the glycerol/ water ratio. We show that the glass transition is extremely broad in temperature for low hydration levels, and that the broadness of $T_{\mathrm{g}}$ is likely due to that several relaxation processes, occurring on different time-scales, contributing to the glass transition. When the hydration level decreases it is suggested that the more local protein relaxations are less slowed downed compared to the global fluctuations, thereby giving rise to a shift of the $T_{\mathrm{g}}$ range to slightly higher temperatures and, in particular, a significant broadening of the transition, in agreement with the calorimetric results.

We also discuss the solvent dynamics and its relation to the glass transition of the protein-solvent system. The results suggest that the cooperative $\alpha$-relaxation of the solvent is involved in the calorimetric glass transition, but that the only observable solvent processes observed at low temperatures for water rich solvents are of too secondary (or local) character to participate in the glass transition. Furthermore, we provide an explanation for why the main solvent process, in such water rich solvents, should be regarded as secondary, and therefore not related to the viscosity, at temperatures below a dynamic crossover occurring at $180 \pm 20 \mathrm{~K}$. A possible explanation, that is consistent with all available relaxation data on hydration water and confined water, for this dynamic crossover is also provided, and it is further discussed how it may affect the glass transition related protein dynamics.

\section{Acknowledgement}

This work was financially supported by the Swedish Foundation for Strategic Research.

\section{References}

[1] J.A. Rupley, G. Careri, Protein hydration and function, Adv. Protein Chem. 41 (1991) 37-172.

[2] J.A. Rupley, P.H. Yang, G. Tollin, in: S.P. Rowland (Ed.), Water in Polymers, American Chemical Society, Washington D.C, 1980.

[3] R. Pethig, Protein-water interactions determined by dielectric methods, Annu. Rev. Phys. Chem. 43 (1992) 177-205.

[4] H. Jansson, J. Swenson, Dynamics of water in molecular sieves by dielectric spectroscopy, Eur. Phys. J. E 12 (2003) S51-S54.

[5] H. Jansson, R. Bergman, J. Swenson, Relation between solvent and protein dynamics as studied by dielectric spectroscopy, J. Phys. Chem. B 109 (2005) 24134-24141.

[6] J. Swenson, H. Jansson, W.S. Howells, S. Longeville, Dynamics of water in a molecular sieve by quasielastic neutron scattering, J. Chem. Phys. 122 (2005) 084505.

[7] J. Swenson, H. Jansson, R. Bergman, Relaxation processes in supercooled confined water and implications for protein dynamics, Phys. Rev. Lett. 96 (2006) 247802 1-4. 
[8] J. Swenson, H. Jansson, J. Hedström, R. Bergman, Properties of hydration water and its role for protein dynamics, J. Phys., Condens. Matter 19 (2007) 205109.

[9] J. Swenson, H. Jansson, R. Bergman, Anomalous behaviour of supercooled wate and its implication for protein dynamics, in: G. Franzese, M. Rubi (Eds.), Aspects of Physical Biology: Biological Water, Protein Solutions, Transport and Replication, Springer-Verlag Berlin, Heidelberger platz 3, D-14197 Berlin, Germany, 2008, pp. 23-42.

[10] M. Vogel, Origins of apparent fragile-to-strong transitions of protein hydration waters, Phys. Rev. Lett. 101 (2008) 225701 1-4.

[11] S. Cerveny, A. Alegria, J. Colmenero, Universal features of water dynamics in solutions of hydrophilic polymers, biopolymers, and small glass-forming materials, Phys. Rev. E (2008) 77.

[12] K.L. Ngai, S. Capaccioli, N. Shinyashiki, The protein "glass" transition and the role of the solvent, J. Phys. Chem. B 112 (2008) 3826-3832.

[13] P.W. Fenimore, H. Frauenfelder, B.H. McMahon, R.D. Young, Bulk-solvent and hydration-shell fluctuations, similar to alpha- and beta-fluctuations in glasses, control protein motions and functions, Proc. Natl. Acad. Sci. U.S.A. 101 (2004) 14408-14413.

[14] H. Frauenfelder, G. Chen, J. Berendzen, P.W. Fenimore, H. Jansson, B.H. McMahon, I. Mihut-Stroe, J. Swenson, R.D. Young, A unified model of protein dynamics, Proc. Natl. Acad. Sci. 106 (2009) 5129-5134.

[15] H. Lichtenegger, W. Doster, T. Kleinert, A. Birk, B. Sepiol, G. Vogl, Heme-solven coupling: a Mossbauer study of myoglobin in sucrose, Biophys. J. 76 (1999) 414-422.

[16] A. Faraone, L. Liu, C.Y. Mou, C.W. Yen, S.H. Chen, Fragile-to-strong liquid transition in deeply supercooled confined water, J. Chem. Phys. 121 (2004) 10843-10846.

[17] H. Frauenfelder, S.G. Sligar, P.G. Wolynes, The energy landscapes and motions of proteins, Science 254 (1991) 1598-1603.

[18] C.A. Angell, Formation of glasses from liquids and biopolymers, Science 267 (1995) 1924-1935.

[19] I.E.T. Iben, D. Braunstein, W. Doster, H. Frauenfelder, M.K. Hong, J.B. Johnson, S. Luck, P. Ormos, A. Schulte, P.J. Steinbach, A.H. Xie, R.D. Young, Glassy behavior of a protein, Phys. Rev. Lett. 62 (1989) 1916-1919.

[20] D. Vitkup, D. Ringe, G.A. Petsko, M. Karplus, Solvent mobility and the protein 'glass' transition, Nat. Struct. Biol. 7 (2000) 34-38.

[21] W. Doster, S. Cusack, W. Petry, Dynamical transition of myoglobin revealed by inelastic neutron-scattering, Nature 337 (1989) 754-756.

[22] W. Doster, The dynamical transition of proteins, concepts and misconceptions, Eur. Biophys. J. Biophys. Lett. 37 (2008) 591-602.
[23] S.R. Elliot, Physics of Amorphous Materials, 2:nd edLongman Scientific and Technical, UK, 1990

[24] H. Nakagawa, Y. Joti, A. Kitao, M. Kataoka, Hydration affects both harmonic and anharmonic nature of protein dynamics, Biophys. J. 95 (2008) 2916-2923.

[25] G. Sartor, A. Hallbrucker, K. Hofer, E. Mayer, Calorimetric glass liquid transition and crystallization behavior of a vitreous, but freezable, water fraction in hydrated methemoglobin, J. Phys. Chem. 96 (1992) 5133-5138.

[26] G.J. Brownsey, T.R. Noel, R. Parker, S.G. Ring, The glass transition behavior of the globular protein bovine serum albumin, Biophys. J. 85 (2003) 3943-3950.

[27] G. Sartor, E. Mayer, G.P. Johari, Calorimetric studies of the kinetic unfreezing of molecular motions in hydrated lysozyme, hemoglobin, and myoglobin, Biophys. J. 66 (1994) 249-258.

[28] Y. Miyazaki, T. Matsuo, H. Suga, Low-temperature heat capacity and glassy behavior of lysozyme crystal, J. Phys. Chem. B 104 (2000) 8044-8052.

[29] W. Doster, A. Bachleitner, R. Dunau, M. Hiebl, E. Luscher, Thermal-properties of water in myoglobin crystals and solutions at subzero temperatures, Biophys. J. 50 (1986) 213-219.

[30] T. Morimoto, T. Iwaki, Dielectric behavior of adsorbed water. 1. Measurement at room-temperature on TiO2, J. Chem. Soc., Faraday Trans. I 83 (1987) 943-956.

[31] M.EV Costa, P.Q Mantas, J.L. Baptista, Effect of electrode alterations on the Ac behavior of Li2O-ZnO humidity sensors, Sens. Actuators, B Chem. 27 (1995) 312-314

[32] R. Richert, Insulated electrodes for eliminating conductivity in dielectric relaxation experiments, Eur. Phys. J. B 68 (2009) 197-200.

[33] R. Bergman, General susceptibility functions for relaxations in disordered systems, J. Appl. Phys. 88 (2000) 1356-1365.

[34] Y. Shibata, A. Kurita, T. Kushida, Real-time observation of conformational fluctuations in $\mathrm{Zn}$-substituted myoglobin by time-resolved transient hole-burning spectroscopy, Biophys. J. 75 (1998) 521-527.

[35] S. Bone, Time-domain reflectometry studies of water binding and structural flexibility in chymotrypsin, Biochim. Biophys. Acta 916 (1987) 128-134.

[36] H. Jansson, F. Kargl, F. Fernandez-Alonso, J. Swenson, Dynamics of a protein and its surrounding environment; a QENS study of myoglobin in water and glycerol mixtures, J. Chem. Phys. 130 (2009) 205101-205113.

[37] S. Khodadadi, S. Pawlus, J.H. Roh, V.G. Sakai, E. Mamontov, A.P. Sokolov, The origin of the dynamic transition in proteins, J. Chem. Phys. 128 (2008) 195106 1-5.

[38] Y. Hayashi, A. Puzenko, Y. Feldman, Slow and fast dynamics in glycerol-water mixtures, J. Non-Cryst. Solids 352 (2006) 4696-4703.

[39] R. Bergman, J. Swenson, Dynamics of supercooled water in confined geometry, Nature 403 (2000) 283-286. 